\title{
Conversion of a Traditional In-Person Feeding Clinic to a Telehealth-Only Model of Care
}

\author{
Sarah E. Fleet ${ }^{1,2}$ (D) $\cdot$ Ryan D. Davidson ${ }^{1,3} \cdot$ Kathleen Carr $^{1} \cdot$ Carolyn Lubenow $^{4} \cdot$ Anna S. Rouse $^{1} \cdot$ Katherine E. Truscott $^{1}$
}

Accepted: 23 November 2021 / Published online: 3 January 2022

(c) The Author(s), under exclusive licence to Springer Science+Business Media, LLC, part of Springer Nature 2021

\begin{abstract}
Purpose: In March 2020, many state, local, and national governments declared various states of emergencies in response to the COVID-19 pandemic. In Massachusetts, where our multidisciplinary pediatric feeding clinic is located, the governor declared of a state of emergency encouraging social distancing, and simultaneously signed an order establishing reimbursement parity for telehealth visits to in-office traditional visits by both commercial and state health insurers. This presented a challenge and an opportunity for our multidisciplinary program for children with pediatric feeding disorders embedded in a large academic children's hospital. In this paper we aim to provide a roadmap for rapid implementation of telehealth practice without a reliance on in-person care in a multidisciplinary pediatric feeding clinic. Description: Within a week, the program pivoted from solely in-person care to $100 \%$ telehealth services for both new and established patients. Through this transition, the program encountered several challenges with technology, scheduling, licensing, and concerns for reinforcing pre-existing healthcare disparities. Assessment: The program quickly overcame many of these challenges and found telehealth to offer benefits to patients such as improved coordination of care with other agencies, reduced appointment times, and reduced travel time and travel cost. Even with a reduction in the number of patients seen per clinic due to the manner in which telehealth was implemented, there was an increase in the number of visits completed with a slight reduction in the no-show rate. Additionally, providers in the program are better able to evaluate feeding practices in the home and understand many of the barriers families may face in implementing interventions. While telehealth does have some challenges, it can help to improve access, communication, and may increase patient satisfaction for children who require multidisciplinary care for their pediatric feeding disorder. Conclusion: Our hope is that billing parity for telehealth will continue to be supported by insurance companies and state governments throughout the remainder of this pandemic, and far beyond.
\end{abstract}

Keywords Pediatric feeding disorder $\cdot$ Multidisciplinary $\cdot$ Telehealth $\cdot$ COVID-19 $\cdot$ Pediatrics

Sarah E. Fleet

sarah.fleet@childrens.harvard.edu

1 Division of Gastroenterology, Hepatology \& Nutrition, Boston Children's Hospital, 300 Longwood Avenue, Boston, MA 02115, USA

2 Department of Pediatrics, Harvard Medical School, 25 Shattuck Street, Boston, MA 02115, USA

3 Department of Psychiatry, Harvard Medical School, 25 Shattuck Street, Boston, MA 02115, USA

4 Feeding and Swallowing Program, Boston Children's Hospital, 300 Longwood Avenue, Boston, MA 02115, USA

\section{Significance}

The use of telehealth for pediatric feeding disorders in a multidisciplinary setting was a growing area in the field, but became commonplace during the novel coronavirus pandemic. Previous research has focused on enhancing care for patients with pediatric feeding disorders using telehealth for specific disciplines, either in conjunction with in-person care or exclusively virtually (Clark et al., 2019; Clawson et al., 2008; Silverman, 2017). However, the implementation of a solely telehealth approach to multidisciplinary pediatric care for both tube-fed and orally fed children with pediatric feeding disorders has not yet been described, nor has it been described as a stand-alone modality for care with a full multidisciplinary team (Medical, Nutrition, Feeding, and Psychosocial supports). This manuscript describes the 
rapid implementation of telehealth in a multidisciplinary clinic for children with feeding disorders as the sole model of care delivery, and the unique benefits and challenges this provided to patients and providers. We believe this experience can inform other multidisciplinary clinics in specialties beyond feeding, which could improve access to care. Furthermore, this experience can also inform preemptive handling of the pitfalls noted in this paper to improve patient experience and patient care.

\section{Purpose}

In this article, we aim to provide a roadmap for rapid implementation of telehealth practice without a reliance on inperson care, in a multidisciplinary pediatric feeding clinic. We aim to share the experience of a clinic without a previous telehealth component, so that other such clinics can improve upon our process, address the pitfalls, and consider implementation of telehealth as a model to improve access to care for patients in pediatric feeding clinics. Though the COVID-19 pandemic was an external force imposed upon the clinic requiring rapid transition to a telehealth model, many aspects of this process would conceivably be the same for other multidisciplinary feeding clinics looking to establish a virtual presence to improve care.

\section{Description of Multidisciplinary Clinic}

The Growth and Nutrition Program is a multidisciplinary clinic at Boston Children's Hospital that specializes in the multidisciplinary evaluation and treatment of children under 7 years of age who have impaired oral intake associated with medical, nutritional, feeding skill, and/or psychosocial dysfunction (Goday et al., 2019). Established in 1984, the Growth and Nutrition Program was one of the first multidisciplinary programs in the country specifically devoted to feeding, malnutrition and poor growth in early childhood. The program has been funded, in part, by a grant from the Massachusetts Department of Public Health, with a goal to address malnutrition in early childhood. The clinic accepts referrals from a wide range of sources-hospital-based or community pediatricians, subspecialists, Early Intervention, Special Supplementation Program for Women, Infants and Children (WIC), and self-referrals.

The team's approach incorporates a biopsychosocial model to promote improved feeding and swallowing, reduction of oral aversion, restoration of nutrition, relief of anxiety, and appropriate family functioning around feeding and mealtimes. Primary providers include gastroenterologists, nurse practitioners, dietitians, speechlanguage pathologists with expertise in feeding, lactation experts, behavioral psychologists, visiting nurses, and social workers. Common diagnoses include prematurity, oral and/or pharyngeal dysphagia, reflux, delay in feeding skills, and Avoidant Restrictive Food Intake Disorder. Other chronic illnesses are often identified during the workup these patients receive and include cow's milk protein allergy, eosinophilic esophagitis, celiac disease, cystic fibrosis, Russell-Silver Syndrome, or other genetic disorders.

\section{Clinic Model Before the COVID-19 Stay-at-Home Order}

Previously, new patients were scheduled as partially-overlapping, in-person, 60-min visits for each discipline, while follow-ups were generally scheduled as separate 30 -min visits with each discipline. There were no telehealth options for patients or providers prior to the COVID-19 pandemic. Patients routinely spent a minimum of $1.5 \mathrm{~h}$ in clinic, with many new patients spending closer to $3 \mathrm{~h}$ in the clinic space. All patients were assigned a primary gastroenterologist or nurse practitioner, dietitian, and feeding therapist to conduct each visit. The psychologist saw patients two-years or older. The program's social worker was also available to provide resources and support to all families during their visit. Visits were often conducted back-to-back, with brief conferencing of clinicians in between, or with some overlap of clinicians in the room to allow for collaboration of care.

Unfortunately, due to unique challenges of the population, late cancellations and no-shows occurred frequently (ranging from 11 to 25\%). This resulted in a large amount of "lost" provider time that could have been allocated to another patient had enough notice been given. There was also a growing burden for administrative staff as the wait list grew to around 4-6 months and maintaining this list while also accommodating new referrals was a challenge.

\section{Impact of the COVID-19 Pandemic}

The COVID-19 pandemic and resultant social distancing measures presented a unique opportunity for healthcare delivery nationwide. Many policy changes at local, state, and national levels impacted the number of patients and providers in the clinic, and mandated that commercial and state health insurers cover healthcare delivery via telehealth with equal reimbursement rates to traditional in-office visits. This allowed telehealth, which may not have been an option for many families in the past, to be a potential mechanism for healthcare delivery. Specifically, within our own institution, hospital policy encouraged our pediatric multidisciplinary clinic to quickly convert visits 
to a telehealth platform, while simultaneously trying to preserve the unique multidisciplinary, collaborative nature of visits.

\section{Use of Telehealth in Pediatric Feeding Populations}

Telehealth has previously been utilized to enhance care for pediatric feeding disorders in varying formats and within several disciplines. Regarding telehealth formats, two different models have been used: "store and forward" where families could record meals or food logs and send to providers, or real time interactions where providers could speak directly to patients and families (Silverman, 2017). Prior to the COVID-19 pandemic, telehealth interventions had been used for delivery of behavioral health components of feeding interventions with high patient satisfaction and treatment effectiveness (Bloomfield et al., 2019; Silverman, 2017). Other models utilized a telehealth enhanced model of care where the initial assessment was conducted in person, but then follow up parent-driven feeding intervention sessions were conducted using telehealth (Clark et al., 2019). In addition to these methods being found to maintain treatment integrity, demonstrate effectiveness, and be satisfactory to families, there was also significant cost benefits (Clark et al., 2019). One clinic utilized telehealth for the full multidisciplinary team, including initial assessment. They found that having patients and families travel to their local clinics or universities to conduct the remote visits was acceptable to families and patients were able to be treated in their home communities (Clawson et al., 2008).

At the time of the transition to a virtual format, literature suggested that telehealth was a viable, safe, and effective process within feeding programs, though there was little literature regarding methods or effectiveness for implementing a purely telehealth model. With this in mind, our program quickly pivoted to an all-telehealth model to ensure patient and provider safety during a global pandemic. Previous research suggested creating a team to identify patient populations for implementation of telehealth, consideration of most appropriate infrastructure, and identification of which telehealth model would fit patient and provider needs (Clawson et al., 2008; Silverman, 2017). However, given the context of the clinic being located in a large academic medical setting and the transition to telehealth occurring in the setting of a global pandemic, some decisions such as which patient groups would be best suited for telehealth within the clinic or clinic decisions around infrastructure were decisions made by hospital leadership. Due to these factors, there were several changes that were made throughout the transition to a purely telehealth model of care.

\section{Description}

\section{Rapid Transition to $100 \%$ Telehealth in Response to COVID-19}

In response to the two state-wide orders in March 2020, the hospital quickly expanded their pre-existing telehealth platform using SBR-Health (a Vidyo-based platform). Though this program had been implemented on a small scale throughout the hospital previously, this specific platform presented several challenges for the Growth and Nutrition Program. The SBR platform only allowed one clinician in a visit; however, up to 5 providers needed to simultaneously meet with the patient at any given time. Initially, other providers joined by phone with the physician/NP completing the visit on SBR-Health and their phone on speaker for all to hear, but this was cumbersome, with poor sound quality and did not allow other providers access to the video component. Many patient families had difficulty downloading the SBR-Health software, and the email with the SBR-Health link and instructions was at times in a language or dialect other than the family's preferred language.

For 2-3 weeks this was the only approved option, but a hospital policy change in mid-April 2020 gave permission to use an encrypted Zoom meeting when more than one provider needed to join. The program immediately shifted to the encrypted Zoom platform, which allowed all clinicians to be present for the same 60-min visit for each patient while maintaining confidentiality for the patient.

To schedule patients using Zoom, the administrative team would call the family with the available appointment dates and times, and once confirmed, would email the Zoom link for the family. All providers were named as hosts so that anyone could start the meeting, and the waiting room was enabled to allow for pre-visit conferencing amongst the care team. Breakout rooms were also enabled so that one provider could continue talking with the patient and family while the rest of the team could confidentially discuss management options and develop a cohesive plan of care before presenting it to the family. Screensharing was also enabled so providers could share growth charts or other parts of the medical record, and so that families could screenshare photos, videos, diet logs or other relevant files with the care team.

With the new telehealth model, visits were conducted in 1-h blocks with all providers present for the visit. Both initial visits and follow up visits were conducted in the same format with psychology having the ability to see families more frequently in between full team visits. Given this new model, each provider had to make changes to the way 
Table 1 Summary of changes in model of care when transitioning from a solely in-person to a purely telehealth visit format

\begin{tabular}{|c|c|c|}
\hline & $100 \%$ in person & $100 \%$ telehealth \\
\hline Visit model & Visits with single provider at a time & All providers in the same visit \\
\hline $\begin{array}{l}\text { Who is in visits (in } \\
\text { addition to provid- } \\
\text { ers) }\end{array}$ & Patient, parent/caregiver, occasionally community providers & $\begin{array}{l}\text { Patient, parent/caregiver, extended family, nanny/daycare } \\
\text { providers, community providers }\end{array}$ \\
\hline Length of visits & $\begin{array}{l}30 \text { min individual visit for each discipline; total of } \\
90-120 \text { min }\end{array}$ & 60 min for multiple disciplines \\
\hline \multicolumn{3}{|l|}{ Disciplines } \\
\hline Gastroenterology & Traditional physical exam by GI provider (MD/NP) & $\begin{array}{l}\text { Exam limited to visual exam, with parent/caregiver con- } \\
\text { ducting other components of physical exam }\end{array}$ \\
\hline Nutrition & Anthropometrics measured on calibrated hospital devices & $\begin{array}{l}\text { Anthropometrics on home scale with calibration or at } \\
\text { primary care office }\end{array}$ \\
\hline Feeding Therapy & $\begin{array}{l}\text { Visual observation of feeding skills and live modeling of } \\
\text { skills in an exam room }\end{array}$ & $\begin{array}{l}\text { Visual observation through zoom of foods offered at home, } \\
\text { in home environment }\end{array}$ \\
\hline Psychology & $\begin{array}{l}\text { Patient present for entirety of evaluation; observation of } \\
\text { behaviors in office setting }\end{array}$ & $\begin{array}{l}\text { Patient present for portions of evaluations; observation of } \\
\text { behaviors in home setting; coached meals in home setting }\end{array}$ \\
\hline Visit frequency & 3 months average & 2 months average \\
\hline
\end{tabular}

in which their assessments were conducted. See Table 1 for a summary of changes in the model of care.

\section{Assessment}

\section{Secondary Changes in Care Model (See Table 1)}

With the transition to telehealth, there were several secondary changes to our care model. The team was now able to accommodate more providers and staff members into the visits, allowing for families to spend less time in a visit. Families were able to invite other providers into the visit with ease, and the team can communicate directly with community workers including those from Department of Children and Families and/or Early Intervention/Birth to 3 during the visit. Nannies, grandparents, and other caregivers were able to join when necessary and multiple parents or guardians can each join the visit.

Previously, follow-up visits in the clinic were often booked in 30-min adjacent time slots. Since families were asked to log-on to a telehealth visit, often with other children and no childcare, visits were scheduled for one hour with all team members present. This reduced provider availability, which was addressed by intermittently adding clinic sessions.

There were changes in the way many providers conducted their portion of the visit. For example, the GI provider, who usually performed a traditional physical exam in the office, was dependent on a visual exam over Zoom, with assistance from the parents who described appearance and palpation of the abdomen. For traditional visits, patients were typically weighed on the same scale every time they came to clinic, and measured on a length board or stadiometer by trained assistants. With the transition to telehealth, families are now asked to weigh and measure their children at home, without any training, and at times, on equipment not meant for children (some families used postage scales, while others used adult standing scales).

When using an in-person care model, clinical feeding evaluations were performed in an exam room, using either parent or clinic-provided snacks, with the child seated in the clinic's feeding chairs or parent's lap. Patient cooperation was a challenge as it could occur at the end of several visits and patient fatigue, hunger, or anxiety were often barriers. Using telehealth, feeding observations are now performed in the patient's own home, in their own feeding seat, and with food from home.

Similarly to the clinical feeding evaluations, child behavior observations as a part of the psychological evaluation and intervention were now conducted in the child's own home. When in-person, the child was present for the entirety of the psychological evaluation, and with telehealth, providers were able to meet with a combination of caregivers individually and caregiver/patient combined. With implementation of telehealth visits, psychology providers were able to also engage family in meal observations and coaching in a natural environment.

Finally, the use of telehealth has allowed many families to follow up more frequently. This is especially true for families who are followed frequently by psychology. Due to the burdens associated with travel, many families were unable to attend visits as often as they would have liked 
or as recommended by our psychologist. With the implementation of telehealth, many families have been able to access this service with more frequency.

\section{Provider Feedback and Visit Numbers}

Throughout the process of transitioning from a 100\% in-person model to a $100 \%$ telehealth model of multidisciplinary care in a pediatric feeding clinic, both providers and patients have provided feedback. One provider noted, "Telehealth has made our care accessible for families who previously could not take time off of work for lengthy appointments or pay for parking. We have been able to follow-up more frequently, and get a direct line of sight into the home feeding environment which has been extraordinarily helpful". Patients have commented on preference for telehealth visits due to decreased length of appointment and decreased need to take time off for themselves or for time out of school for their children. Many have also noted that they feel safer not coming to the hospital for visits, in this pandemic era.

In addition to patient and provider feedback, there were also changes in the number of visits scheduled and completed. The change in structure of visits impacted the number of visits that were able to be scheduled. Prior to transition, most providers would be able to see 5-6 patients per clinic; however, with transition to telehealth and change to all providers meeting with family simultaneously, providers were able to see 4 patients per clinic. Though there were fewer available appointments, the number of completed visits increased by $22 \%$ while there were a similar number of unique patients seen in the clinic. In addition, the number of no-show or late cancellation visits remained stable even in the setting of significant changes in routines for families.

\section{Conclusion}

\section{Benefits to Telehealth in a Pediatric Multidisciplinary Clinic}

Telehealth drastically altered care delivery to patients with pediatric feeding disorders. Providers noticed improvements in feeding evaluations, medication reconciliation, diet history, and communication with community providers. The shift to telehealth also allowed for the reallocation of services within the Growth and Nutrition Program. Further, some barriers to care, such as distance and time required to travel to appointments, have improved, allowing more frequent follow-ups for some patients.

With the shorter visit times and ability to engage in feeding and behavioral evaluations in the home setting, providers are able to conduct observations in a more natural setting. Patients are well-rested with shorter visit times and less anxious since they are in their home environment. This has reduced the need for home visits from our visiting nurse, who now uses the time to support the program in other ways, including care coordination with other providers and with the school, and responding to urgent calls. When children return to school, the visiting nurse will be able to direct efforts to in-school observations, as in-home feeding observations will continue to be done remotely.

Additionally, reconciliation of medications, vitamins, and diet recall are now much easier. Families can show medication bottles, vitamin containers, or packaged foods to providers directly. In addition to improving the efficiency of this process, it has also shed light on several medication errors. Dietitians are now able to directly observe the nutrition facts on the package and obtain necessary macro- and micronutrient data that was previously only obtainable by lengthy Google Image searches. In some instances, this has resulted in dietitians recognizing allergenic foods that were unknowingly being offered.

With the ability of more family members, caregivers, and providers to be present for visits, it allows for improved data gathering and history taking, as well as a better understanding of improvements and challenges for patients. Further, the clinic social worker is able to directly visualize the home, and better identify barriers to care. Overall, the social component of our biopsychosocial model has expanded immensely, and video-based telehealth allows improved family-centered care.

The Growth and Nutrition Program accepts referrals from in- and out-of-state, as well as internationally. The distance many families travel to the Program parlays a significant time and financial burden. The use of telehealth has allowed many families from across the state to follow up more frequently since it eliminates the need to travel to appointments. This was a significant contributor to family's ability to participate in telehealth visits more frequently as well as increasing the number of clinic visits, even with less clinic time available.

\section{Challenges to Pure Telehealth Model for Pediatric Multidisciplinary Clinic}

Though there are many benefits to using telehealth, this transition has not been without its struggles. Initially, the transition period posed challenges with the technology since the hospital-supported platform only allowed one clinician at a time. This was not useful for our typical practice model with multiple providers present, but once visits were switched to the Zoom platform, group visits could be accommodated. There were also scheduling challenges. Most of the providers made scheduling accommodations, by adding clinic sessions on other days of the week, or by extending their clinic hours to meet the demand. The clinic 
began to implement shorter follow-up appointments for patients that do not need the full hour, freeing up providers to see more patients per clinic session. However, providers reported quickly that the shorter time may not be sufficient as visits may run long and providers struggle to address all of the facets of the care plan in the shorter visit. This significant shift in scheduling created additional burdens and change in workflow for the administrative staff. Separate Zoom meetings needed to be set up for each patient appointment and then emailed to patients and providers in addition to typical workflow. Administrative staff also field a large volume of calls from patients who cannot find the Zoom link in their email or are having trouble logging on.

One of the largest early issues facing the clinic were licensing and billing restrictions for each type of provider. In the beginning of the transition to telehealth services, some providers (physicians and psychologist) were able to see in-state patients immediately, while others had to wait for additional guidance from licensing agencies. Licensing remains an ever present controversy, as it continues to evolve through the pandemic, and the landscape is different month-to-month, state-by-state, and disciplineto-discipline. Successful implementation of a $100 \%$ telehealth clinic requires assistance from billing, compliance and legal departments, and frequently updated guidance regarding this.

As we initially transitioned to a purely telehealth model, there were significant concerns that many patients would be unable to join a telehealth visit due to lack of highquality internet access; however, this does not seem to be the case. Very few families have opted out of telehealth visits, and for the few families that did, a telephone call was offered and the visit was conducted by phone. Some of these families have since switched to telehealth visits. The hospital also implemented programs to provide families with frequent medical appointments access to technology and high-quality internet access. In addition, there were also concerns about non-English speaking families and their ability to access the instructions in the email with the Zoom link. Hospital interpreters now call families the day before a visit in their preferred language to help walk them through the log-in process and join the Zoom call for the appointment. If a non-English speaking patient does not $\log$ on to the visit promptly, the interpreter calls them and helps them log on.

Finally, the lack of accurate anthropometrics has been a challenge in the feeding program, as average weight gain, weight for age, and weight for length z-scores are benchmarks for patient progress. Xxx Hospital quickly drafted an illustrated instruction guide on how to weigh and measure a child's length, height, and head circumference which is sent with the Zoom meeting invite, asking families to take two measurements on the same scale a few weeks apart in order to calculate a weight gain velocity, and to account for the differences between their scale and the clinic's. We also partnered with pediatrician offices closer to patients' homes, who were able to obtain height and weight measurements and communicate them to our team.

\section{Future Implications for Practice and Future Directions}

Ultimately, telehealth has revolutionized how we provide care in our multidisciplinary clinic for young children with pediatric feeding disorders. Patients continue to provide feedback that they prefer telehealth visits and would prefer not to travel to clinic locations in the future. As the clinic continues to adapt to the changing landscape of medicine, it is expected that telehealth practices will continue.

Over time, as billing, licensing, and ethical guidelines continue to change and develop, it will be important for clinics and providers to remain well-versed in most up-to-date ethical practices as well as standards of care utilizing telehealth models. This may include continued modifications to telehealth visit infrastructure and methods by which telehealth is conducted by various providers. Since our program transitioned to a purely telehealth model, there has been a rapidly growing literature on the effectiveness of various models of telehealth implementation for pediatric feeding clinics. Similar to research available prior to the pandemic, telehealth in pediatric feeding has been found to be acceptable for families, (Peterson et al., 2020), allow for continued integrity of behavioral interventions when implemented by parents (Andersen et al., 2021), and effective treatment outcomes (Peterson et al., 2020).

Moving forward, a mixed model of in-person and virtual visits is expected, with about $75 \%$ of care delivery continuing to be done virtually within our clinic. The remaining $25 \%$ of visits, which are to be held in person, will still use a mixed model to accommodate social distancing standards as we continue to struggle to anticipate the "new normal". There will be one in-person clinician-either the physician or the feeding therapist depending on need-and the rest of the team will join the visit by Zoom, using the computer in the exam room in clinic. The hope is to maintain the group visit model, but allow for accurate anthropometrics, physical exam, and/or in-person clinical feeding evaluation when deemed necessary. 


\section{Summary}

\section{Broader Implications for Healthcare Delivery and Policy}

The COVID-19 pandemic required an immediate pivot in the way clinicians provide care to their patients. To reduce the spread of the virus and for the safety of patients and staff, telehealth was swiftly and completely adopted by all facets of the healthcare system. While it initially presented challenges specific to a multidisciplinary clinic with multiple providers and the need for an integrated care plan, once those challenges were addressed, the use of telehealth has improved access for patients and their families. While there were initial concerns that telehealth could enhance healthcare disparities, in this population, the use of telehealth has seemingly reduced barriers to care, and allowed patients to receive the quality of care they require during this unprecedented time. Telehealth has reduced the travel, time, and financial burden of in-person clinic visits, and has given providers unique insight into patients' lives, and homes. Thus far, telehealth has been patient and family-centered, efficient for group visits, and an acceptable alternative to in-person visits; acceptable solutions for some of the potential drawbacks which have been identified. Our hope is that billing parity will continue to be supported by insurance companies and state governments, and that this will become a priority at the national level far beyond the remainder of this pandemic.

Author Contributions SEF: article development, writing, editing, revisions; RDD: writing, editing, revisions; KC: content suggestions, editing; CL: content suggestions, editing; AR: content suggestions, editing; KT: content suggestions, editing.

Funding Not applicable.

Data Availability Not applicable.

Code Availability Not applicable.

\section{Declarations}

Conflict of interest The authors declare that they have no conflict of interest.
Ethical Approval Not applicable.

Consent to Participate Not applicable.

Consent for Publication Not applicable.

\section{References}

Andersen, A. S., Hansen, B. A., Hathaway, K. L., \& Elson, L. A. (2021). A demonstration of caregiver-implemented functional analysis of inappropriate mealtime behavior via telehealth. Behavior Analysis in Practice, 14, 1067-1072.

Bloomfield, B. S., Fischer, A. J., Clark, R. R., \& Dove, M. B. (2019). Treatment of food selectivity in a child with Avoidant/Restrictive Food Intake Disorder through parent teleconsultation. Behavior Analysis in Practice, 12, 33-43.

Clark, R. R., Fischer, A. J., Lehman, E. L., \& Bloomfield, B. S. (2019). Developing and implementing a telehealth enhanced interdisciplinary pediatric feeding disorders clinic: A program description and evaluation. Journal of Developmental and Physical Disabilities, 31, 171-188.

Clawson, B., Selden, M., Lacks, M., Deaton, A. V., Hall, B., \& Bach, R. (2008). Complex pediatric feeding disorders: Using teleconferencing technology to improve access to a treatment program. Pediatric Nursing, 34(3), 213-216.

Goday, P. S., Huh, S. Y., Silverman, A., Lukens, C. T., Dodrill, P., Cohen, S. S., Delaney, A. L., Feuling, M. B., Noel, R. J., Gisel, E., Kenzer, A., Kraus de Camargo, O., Browne, J., \& Phalen, J. A. (2019). Pediatric feeding disorder: Consensus definition and conceptual framework. Journal of Pediatric Gastroenterology and Nutrition, 68, 124-129.

Peterson, K. M., Ibanez, V. F., Volkert, V. M., Zeleny, J. R., Engler, C. W., \& Piazza, C. C. (2020). Using telehealth to provide outpatient follow-up to children with avoidant/restrictive food intake disorder. Journal of Applied Behavior Analysis, 9999, 1-19.

Silverman, A. (2017). Telehealth interventions for feeding problems. In S. Angel (Ed.), Feeding problems in children (pp. 261-276). Boca Raton: CRC Press.

Publisher's Note Springer Nature remains neutral with regard to jurisdictional claims in published maps and institutional affiliations. 